\title{
TYPE RADICALS AND QUASI-DECOMPOSITIONS OF TORSION-FREE ABELIAN GROUPS
}

\author{
OTTO MUTZBAUER
}

(Received 8 March 1990; revised 11 May 1990)

Communicated by $\mathrm{H}$. Lausch

\begin{abstract}
A composition sequence for a torsion-free abelian group $A$ is an increasing sequence of pure subgroups with rank 1 quotients and union $A$. Properties of $A$ can be described by the sequence of types of these quotients. For example, if $A$ is uniform, that is all the types in some sequence are equal, then $A$ is complete decomposable if it is homogeneous. If $A$ has finite rank and all permutations of one of its type sequences can be realized, then $A$ is quasi-isomorphic to a direct sum of uniform groups.
\end{abstract}

1991 Mathematics subject classification (Amer. Math. Soc.): $20 \mathrm{~K} \mathrm{15,} 20 \mathrm{~K} 20$.

\section{Type radical}

In [M1] type chains are defined. We extend this concept to torsion-free abelian groups of infinite rank.

An ascending sequence of pure subgroups in a torsion-free abelian group not necessarily of finite rank with ranks increasing by 1 is called a composition sequence of $A$ :

$$
0=A_{1} \stackrel{t_{1}}{\subset_{*}} A_{2} \stackrel{t_{2}}{\complement_{*}} \cdots \subset_{*} A_{\beta} \stackrel{t_{\beta}}{\subset_{*}} A_{\beta+1} \stackrel{t_{\beta+1}}{\subset}{ }_{*} \cdots \subset_{*} A=A_{\alpha}
$$

where $C_{*}$ indicates a pure subgroup and $\alpha$ is an ordinal number whose cardinality is equal to the rank of $A$ and $A_{\beta}=\bigcup_{i<\beta} A_{i}$ if $\beta$ is a limit ordinal. The quotients $A_{i+1} / A_{i}$ are rational groups of type $t_{i}$. The sequence $\left(t_{i} \mid i<\alpha\right)$ is called the corresponding type sequence. A torsion-free abelian

(C) 1992 Australian Mathematical Society 0263-6115/92 \$A2.00+0.00 
group is said to be radical or more precisely $t$-radical if there is a type $t$ and a type sequence $\left(t_{i} \mid i<\alpha\right)$ with $t_{i} \geq t$ for all $i$. Following a very well known concept in non-abelian groups we define for a torsion-free abelian group $A$ iterated type subgroups-like iterated centers in the non-abelian case, for instance [R; Section 1.3]-for all $i<\alpha$ by $A^{1}(t)=A(t)=\{a \in$ $A \mid t(a) \geq t\}$,

$$
A^{i+1}(t) / A^{i}(t)=\left(A / A^{i}(t)\right)(t)
$$

and $A^{\beta}(t)=\bigcup_{i<\beta} A^{i}(t)$ if $\beta$ is a limit ordinal. The union $A^{\infty}(t)=\bigcup_{i<\alpha} A^{i}(t)$ is called the $t$-radical or simple the radical of $A$. The subgroups $A^{i}(t)$ and $A^{\infty}(t)$ are obviously fully invariant and pure. By analogy with the notations used for non-abelian groups [R; Section 1.3] the $t$-radical $A^{\infty}(t)$ would be more consistently denoted hyper-t-subgroup; but we prove in Theorem 1.4 that $A^{\infty}(t)$ is a typical radical.

P. Schultz [S] defined in 1978 a $T$-sequence which contains this type radical as a special case.

EXAMPLE. There is a torsion-free abelian group $A$ having a sequence of iterated type subgroups $A^{i}(t)$ of length $\omega+1$. Let $p_{1}, p_{1}, \ldots$ be the sequence of all primes, let

$$
B=\left\langle x_{i}, p_{j}^{-(i+1)}\left(x_{1}+p_{j} x_{2}+\cdots+p_{j}^{i} x_{i+1}\right) \mid 1 \leq i, j<\infty\right\rangle \subset \bigoplus_{i=1}^{\infty} \mathbf{Q} x_{i} .
$$

Let $(1,1, \ldots) \in t$ and $B_{i}=\left\langle x_{1}, \ldots, x_{i}\right\rangle_{*}^{B}$ the pure subgroup of $B$ generated by $\left\langle x_{1}, \ldots, x_{i}\right\rangle$. A composition sequence of $B$ is

$$
0=B_{0} \subset_{*} B_{1} \subset_{*} B_{2} \subset_{*} \cdots \subset_{*} B=\bigcup_{i=1}^{\infty} B_{i}
$$

and $t\left(B_{i} / B_{i-1}\right)=t$, that is, all $B_{i}$ and $B$ are radical groups having a type sequence where all types are equal to $t$. Such groups will later be called uniform or $t$-uniform. Furthermore $B^{i}(t)=B_{i}$ and $B / B_{i} \cong B$ for all $i$. Therefore $B^{\omega}(t)=\bigcup_{i=1}^{\infty} B^{i}(t)=\bigcup_{i=1}^{\infty} B_{i}=B$. Let

$$
A=\left\langle B, y, p_{i}^{-1}\left(y+x_{i}\right) \mid 1 \leq i<\infty\right\rangle \subset \mathbf{Q} y \oplus \bigoplus_{i=1}^{\infty} \mathbf{Q} x_{i} .
$$

Then $B$ is pure in $A$ and $t(A / B)=t$, because $A / B=\langle y+B\rangle_{*}$. Thus $A^{\omega+1}(t)=A$. Moreover $A$ is a $t$-uniform group (see Section 2) and the subgroups $B_{i}=A^{i}(t)$ are strongly indecomposable $t$-uniform groups of rank $i$. Let especially $\mathbf{E}=B_{2}$ for later use. A similar example of rank 2 can also be found in $[\mathrm{G}]$.

We collect some essential properties of radical groups. 
Proposition 1.1. A torsion-free abelian group $A$ is a $t$-radical group if and only if it equals its $t$-radical, that is, $A=A^{\infty}(t)$.

Proof. If $A=A^{\infty}(t)$, then by definition of the iterated type subgroups a composition sequence comprising these subgroups has quotients $A_{i+1} / A_{i}$ with types $\geq t$. Conversely let $A$ be a $t$-radical group, that is, there is a composition sequence

$$
0=A_{1}{\stackrel{t_{1}}{\subset}}_{*} A_{2}{\stackrel{t_{2}}{\subset}}_{*} \cdots \subset_{*} A_{\alpha}=A
$$

with types $t_{1}=t\left(A_{i+1} / A_{i}\right) \geq t$. By transfinite induction on $i$ we show that for all $i<\alpha, A_{i} \subseteq A^{\infty}(t)$. When $i=1$ the result is trivial. Assume now $A_{i} \subseteq A^{\infty}(t)$. Thus

$$
\frac{A_{i+1}+A^{\infty}(t)}{A_{i}+A^{\infty}(t)}=\frac{A_{i+1}+A^{\infty}(t)}{A^{\infty}(t)}
$$

is either 0 , if $A_{i+1} \subset A^{\infty}(t)$, or isomorphic to $A_{i+1} / A_{i}$, that is, of type $t_{i} \geq t$. Consequently $\left(A_{i+1}+A^{\infty}(t)\right) / A^{\infty}(t) \subset\left(A / A^{\infty}(t)\right)(t)=0$ and $A_{i+1} \subset A^{\infty}(t)$. If $\beta$ is a limit ordinal, then by hypothesis $A_{k} \subset A^{\infty}(t)$ if $k<\beta$ and $A_{\beta}=\bigcup_{k<\beta} A_{k} \subset A^{\infty}(t)$.

Proposition 1.2. The pure hull of a t-radical subgroup in a torsion-free abelian group is $t$-radical and extensions of two t-radical groups are $t$-radical.

Proof. Extensions of two $t$-radical groups are obviously $t$-radical. To prove the first statement let $B$ be a $t$-radical subgroup of $A$, that is, there is a composition sequence

$$
0=B_{1} \stackrel{t_{1}}{\complement_{*}} B_{2}{\stackrel{t_{2}}{\subset}}_{*} \cdots \subset_{*} B_{\beta} \stackrel{t_{\beta}}{\complement_{*}} B_{\beta+1} \stackrel{t_{\beta+1}}{\complement} \ldots \subset_{*} B=B_{\alpha}
$$

with type sequence $\left(t_{\beta} \mid \beta<\alpha\right)$ and $t_{\beta} \geq t$ for all $\beta<\alpha$. Let $C_{\beta}=\left\langle B_{\beta}\right\rangle_{*}^{A}$ and $C=B_{*}^{A}$ be the pure hulls of $B_{\beta}$ and $B$ in $A$. It is enough to prove that all quotients of the sequence $\left(C_{\beta} \mid \beta<\alpha\right)$ are of type $\geq t$. But

$$
B_{\beta+1} / B_{\beta}=\left(C_{\beta+1} \cap B\right) /\left(C_{\beta} \cap B\right) \tilde{\subset} C_{\beta+1} / C_{\beta},
$$

so $C=B_{*}^{A}$ is a $t$-radical group.

Proposition 1.3. Torsion-free homomorphic images of t-radical groups and arbitrary sums of $t$-radical subgroups are $t$-radical.

Proof. Let $B$ be a pure subgroup of the $t$-radical group $A$. Let

$$
0=A_{1}{\stackrel{t}{C_{1}}}_{*} A_{2}{\stackrel{t_{2}}{\subset}}_{*} \cdots \subset_{*} A_{\beta}{\stackrel{t_{\beta}}{\subset}}_{*} \cdots \subset_{*} A=A_{\alpha}
$$


be a composition sequence with rational quotients $A_{i+1} / A_{i}$ of type $\geq t$. Let $C_{i}=\left\langle A_{i}+B\right\rangle_{*}^{A}$, so $C_{i} / B=\left\langle\left(A_{i}+B\right) / B\right\rangle_{*}^{A / B}$. The quotient $\left(A_{i+1}+B\right) /\left(A_{i}+B\right)$ is either a torsion group, that is $C_{i}=C_{i+1}$, or a torsion-free homomorphic image of $A_{i+1} / A_{i}$, hence isomorphic to $A_{i+1} / A_{i}$. Then, as in the proof of 1.2 we have $A_{i+1} / A_{i} \tilde{\subset} C_{i+1} / C_{i}$. Omitting equalities in the sequence

$$
0 \subset C_{1} / B \subset C_{2} / B \subset \cdots \subset A / B
$$

we get a composition sequence of $A / B$ with rational quotients of type $\geq t$, so $A / B$ is $t$-radical.

To prove the second statement let $\left(B_{\beta} \mid \beta<\alpha\right)$ be a well-ordering of a set of $t$-radical subgroups (not necessarily pure) in the torsion-free abelian group $A$. Let $C_{\beta}=\sum_{\gamma<\beta} B_{\gamma}$. Consider the sequence

$$
0 \subset C_{1} \subset C_{2} \subset \cdots \subset C_{\beta} \subset C_{\beta+1} \subset \cdots \subset \sum_{\beta<\alpha} B_{\beta}=C .
$$

We prove by transfinite induction that $C$ is $t$-radical. Certainly $C_{1}=B_{1}$ is $t$-radical. Assume $C_{i}$ to be $t$-radical, then the pure hull $D=\left\langle C_{i}\right\rangle_{*}^{C_{i+1}}$ is $t$-radical by the first statement and

$$
C_{i+1} / D=\left(C_{i}+B_{i+1}\right) / D=\left(D+B_{i+1}\right) / D \cong B_{i+1} /\left(B_{i+1} \cap D\right)
$$

is a torsion-free homomorphic image of the $t$-radical group $B_{i+1}$, hence $t$ radical by 1.1. Thus $C_{i+1}$ is the extension of two $t$-radical groups and again $t$-radical by 1.2. If $\beta$ is a limit ordinal, then $C_{\beta}=\bigcup_{\gamma<\beta} C_{\gamma}$ and by 1.2 we may assume all $C_{\gamma}$ to be pure $t$-radical subgroups of $C_{\beta}$, that is, $C_{\gamma+1} / C_{\gamma}$ is $t$-radical by the first statement. Hence $C_{\beta}$ is $t$-radical. Eventually $C$ is $t$-radical.

By 1.3 and 1.4 the class of $t$-radical groups is a radical class in the classical sense [R; Section 1.3]; and the $t$-radical is a radical as usual by the following result.

TheOREM 1.4. The $t$-radical $A^{\infty}(t)$ of $A$ is the sum of all $t$-radical subgroups of $A$ and is itself the unique maximal $t$-radical subgroup. The $t$-radical of the quotient $A / A^{\infty}(t)$ is 0 .

Proof. Let $B$ be a (not necessarily pure) $t$-radical subgroup of $A$, that is, $B$ has a composition sequence

$$
0=B_{1} \stackrel{t}{C}_{*} B_{2}{\stackrel{t_{2}}{C}}_{*} \cdots \subset_{*} B_{\alpha}=B
$$

with corresponding type sequence $\left(t_{i} \mid i<\alpha\right)$, where $t_{i}=t\left(B_{i+1} / B_{i}\right) \geq t$. We prove by transfinite induction that $B_{i} \subset A^{\infty}(t)$ for all $i<\alpha$. 

then

When $i=1$ the result is trivial. Assume $B_{i} \subset A^{\infty}(t)$. If $x \in B_{i+1} \backslash B_{i}$,

$$
t \leq t^{B_{i+1} / B_{i}}\left(x+B_{i}\right) \leq t^{A / A^{\infty}(t)}\left(x+A^{\infty}(t)\right),
$$

hence $x \in A^{\infty}(t)$ and $B_{i+1} \subset A^{\infty}(t)$. If $\beta$ is a limit ordinal and $B_{k} \subset A^{\infty}(t)$ for all $k<\beta$, then $B_{\beta}=\bigcup_{k<\beta} B_{k} \subset A^{\infty}(t)$, and all $t$-radical subgroups of $A$ are contained in the $t$-radical $A^{\infty}(t)$ of $A$ which is also a $t$-radical group. Thus the $t$-radical $A^{\infty}(t)$ is the unique maximal $t$-radical subgroup, and the quotient $A / A^{\infty}(t)$ has $t$-radical 0 , because otherwise we would get a contradiction to the maximality of $A^{\infty}(t)$ by 1.2 .

ReMARK. For a pure subgroup $B$ of a torsion-free abelian group $A$ we have obviously $B(t)=B \cap A(t)$ for all types $t$; but for iterated type subgroups we have only $B^{i}(t) \subset A^{i}(t)$ and $B^{\infty}(t) \subset A^{\infty}(t)$ in general. This will be proved by transfinite induction on $i$. The case $i=1$ is clear. If $B^{i}(t) \subset A^{i}(t)$ and $x \in B^{i+1}(t)$, then

$$
t^{A / A^{i}(t)}\left(x+A^{i}(t)\right) \geq t^{A / B^{i}(t)}\left(x+B^{i}(t)\right)=t^{B / B^{i}(t)}\left(x+B^{i}(t)\right) \geq t,
$$

that is, $x \in A^{i+1}(t)$. If $\beta$ is a limit ordinal and $B^{k}(t) \subset A^{k}(t)$ for all $k<\beta$, then

$$
B^{\beta}(t)=\bigcup_{k<\beta} B^{k}(t) \subset \bigcup_{k<\beta} A^{k}(t)=A^{\beta}(t) .
$$

Equality does not hold in general. In the group

$$
\left.\mathbf{E}=\left\langle x_{1}, x_{2}, p^{-2}\left(x_{1}+p x_{2}\right)\right| p \text { prime }\right\rangle \subset \mathbf{Q} x_{1} \oplus \mathbf{Q} x_{2}
$$

we take the pure subgroup $\mathrm{Z} \cong C=\left\langle x_{2}\right\rangle \subset_{*} \mathbf{E}$, using the notation of the first example. With $(1,1, \ldots) \in t$ we have $\mathbf{E}=\mathbf{E}^{2}(t)=\mathbf{E}^{\infty}(t)$ but $C^{\infty}(t)=$ $C(t)=0$, hence

$$
0=C^{\infty}(t)=C^{2}(t) \neq C=C \cap \mathbf{E}^{2}(t)=C \cap \mathbf{E}^{\infty}(t) .
$$

The intersection of the $t$-radical and the $s$-radical certainly contains the $t \cup s$-radical, $A^{\infty}(s) \cap A^{\infty}(t) \supset A^{\infty}(s \cup t)$, but in general we do not have equality as the following example shows. Let

$$
A=\left\langle x, y, z, p^{-2}(x+p z), q^{-2}(y+q z) \mid p \in P, q \in Q\right\rangle \subset \mathbf{Q} x \oplus \mathbf{Q} y \oplus \mathbf{Q} z,
$$

where $P$ and $Q$ are two infinite disjoint sets of primes. For the types of $x$ and $y$ we have $t=t(x)=t\left(\left\langle p^{-1} \mid p \in P\right\rangle\right)$ and $s=t(y)=t\left(\left\langle q^{-1} \mid q \in Q\right\rangle\right)$ respectively. We have

$$
\begin{gathered}
A^{\infty}(t)=A^{2}(t)=\langle x, z\rangle_{*}, \quad A^{\infty}(s)=A^{2}(s)=\langle y, z\rangle_{*}, \\
A^{\infty}(t) \cap A^{\infty}(s)=\langle z\rangle_{*} \cong \mathbf{Z}
\end{gathered}
$$

but $A^{\infty}(t \cup s)=A(t \cup s)=0$. 
The intersection of $t$-radical or even $t$-uniform subgroups is in general not $t$-radical as the following example shows. Let

$$
\left.A=\left\langle x, y, z, p^{-2}(x+p z), p^{-2}(y+p z)\right| p \text { prime }\right\rangle \subset \mathbf{Q} x \oplus \mathbf{Q} y \oplus \mathbf{Q} z .
$$

We have, using the notation of the first example, that the subgroups $\langle x, z\rangle_{*} \cong$ $\langle y, z\rangle_{*} \cong \mathbf{E}$ are $t$-uniform where $(1,1, \ldots) \in t$; but their intersection is $\langle z\rangle_{*} \cong \mathbf{Z}$ and not $t$-radical.

Proposition 1.5. The t-radical of a torsion-free abelian group $A$ equals the intersection of all pure subgroups $R$ such that $A / R$ has $t$-radical 0.

Proof. By 1.4 the $t$-radical of $A / A^{\infty}(t)$ is 0 . We have to show that $A^{\infty}(t) \subset R$ is $A / R$ has trivial $t$-radical. Assume this to be false. Then there is a first ordinal $i$ such that $A^{i}(t) \not \subset R$. Clearly $i$ is not a limit ordinal, so $A^{i-1}(t) \subset R$. By the definition of $A^{i}(t)$, there exists a subgroup $S$ of $A$ such that $S / A^{i-1}(t)$ is a $t$-radical subgroup of $A / A^{i-1}(t)$ and $S \not \subset R$. Now torsion-free homomorphic images of $t$-radical groups are $t$-radical by 1.3 , so we may apply the natural homomorphism of $A / A^{i-1}(t)$ onto $A / R$, concluding that $S R / R$ is a $t$-radical subgroup of $A / R$. But this implies that $S R / R$ lies in the $t$-radical of $A / R$ by 1.4 , which is trivial. Thus $S \subset R$, a contradiction.

\section{Uniform groups}

A torsion-free abelian group with uniform type sequence $\left(t_{i} \mid i<\alpha\right)$ with $t_{i}=t$ for all $i$ is called uniform or more precisely $t$-uniform. A $t$-uniform group is a special $t$-radical group and equals its $t$-radical by 1.1 . The example $B$ in Section 1 shows that there are uniform groups of arbitrary finite rank which are strongly indecomposable. Moreover uniformity is not inherited by pure subgroups and torsion-free homomorphic images as the example $\mathbf{E}$ shows with $\left\langle x_{2}\right\rangle_{*}^{\mathrm{E}}$ and $\mathbf{E} /\left\langle x_{2}\right\rangle_{*}$ respectively.

Uniformity together with some other properties reduces to complete decomposability.

Proposition 2.1. For a uniform group $A$ the following are equivalent:

(1) $A$ is completely decomposable,

(2) $A$ is separable,

(3) $A$ is homogeneous. 
In this case $A$ is completely decomposable homogeneous.

Proof. That (1) implies (2) is trivial. To show that (3) is a consequence of (2), let $A$ be separable with $t$-uniform composition sequence $0 \subset_{*} A_{1} \subset_{*}$ $\cdots C_{*} A$. We prove by transfinite induction that all $A_{i}$ are homogeneous of type $t$. The case $i=1$ is trivial. Assume $A_{i}$ to be homogeneous of type $t$. This and the $t$-uniformity of $A$ imply that all elements $a \in A_{i+1} \backslash A_{i}$ have the same type $t(a)=s \leq t$. We have to show $t(a)=t$. So we may assume $t(a)<t$. Let $B$ be a completely decomposable direct summand of $A$ of finite rank containing $a$. Say, $A=B \oplus C$, hence $A(t)=B(t) \oplus C(t)$ and $(B+A(t)) / A(t)$ is a direct summand of $A / A(t)$ which implies $B+A(t)$ to be pure in $A$. We have that $B(t)=A(t) \cap B$ is a direct summand of $B$. Thus $B+A(t)=A(t) \oplus D$ where $D \neq 0$ and all types of $D$ are strictly less than $t$. By purity $B+A(t)$ contains $A_{i+1}=\left\langle A_{i}, a\right\rangle_{*}$. Further, $A_{i+1} \not \subset A(t)$ hence $A_{i+1} \cap A(t)=A_{i}$ and we obtain the contradiction

$$
A_{i+1} / A_{i} \cong \frac{A(t)+A_{i+1}}{A(t)} \subset \frac{A(t) \oplus D}{A(t)} \cong D
$$

by $t\left(A_{i+1} / A_{i}\right)=t$. So $A_{i+1}$ is homogeneous of type $t$. If $\beta$ is a limit ordinal, then $A_{\beta}=\bigcup_{\alpha<\beta} A_{\alpha}$ is the union of pure homogeneous subgroups of type $t$ and therefore homogeneous of type $t$ as required.

We now prove that (3) implies (1). If

$$
0=A_{0} \subset_{*} A_{1} \subset_{*} A_{2} \subset \cdots \subset_{*} A_{\alpha}=A
$$

is a $t$-uniform composition sequence of $A$, then for all ordinals $\beta<\alpha$ we have that $A_{\beta+1} / A_{\beta}$ is a rational group of type $t$ and by homogeneity of $A$ all elements in $A_{\beta+1} \backslash A_{\beta}$ are of type $t$. Hence by the lemma of Baer [F; 86.5] $A_{\beta}$ is a direct summand of $A_{\beta+1}$ and $A_{\beta+1}=\left\langle a_{\beta+1}\right\rangle_{*}^{A} \oplus A_{\beta}$. The rational groups $\left\langle a_{\beta}\right\rangle_{*}^{B}, \beta<\alpha$ generate their direct sum $\bigoplus_{\beta<\alpha}\left\langle a_{\beta}\right\rangle_{*}^{A}$ which equals $A=\bigcup_{\beta<\alpha} A_{\beta}$, and $A$ is completely decomposable.

EXAMPLE. The Baer-Specker group $B=Z^{\aleph_{0}}$ is an uncountable, homogeneous group which is $\aleph_{1}$-free but not free [F; 19.2], that is, not completely decomposable and hence not uniform.

The following frequently used fact has a simple proof.

LEMMA 2.2. If $t$ is a type in the typeset of $A$ and if $\left(t_{i} \mid i<\alpha\right)$ is a type sequence of $A$, then there is an $i$ with $t \leq t_{i}$.

Proof. Let

$$
0=A_{1}{\stackrel{t_{1}}{\subset}}_{*} A_{2}{\stackrel{t_{2}}{\subset}}_{*} \cdots \subset_{*} A_{\alpha}=A
$$


be the corresponding composition sequence. If $x \in A$ is an element of type $t(x)=t$, then there is an $i$ such that $x \in A_{i+1}$ but $x \notin A_{i}$, hence

$$
t_{i}=t\left(A_{i+1} / A_{i}\right)=t^{A_{i+1} / A_{i}}\left(x+A_{i}\right) \geq t^{A}(x)=t .
$$

REMARK. Let $A$ be a torsion-free abelian group of finite rank with pure subgroup $B$ of corank 1 . Then for all elements $x \in A \backslash B, t(x) \cap I T(B)=$ $I T(A)$. All elements $x \in A \backslash B$ with $t(x) \leq I T(B)$ have the same type $t(x)=I T(A) \leq I T(B)$. Hence if $A$ is a $t$-uniform group of finite rank with a pure completely decomposable homogeneous subgroup of type $t$ and corank 1, then all $x \in A \backslash B$ have the same type $t(x)=I T(A) \leq t$ and the typeset of $A$ has only the types $I T(A)$ and $t$. In particular, the typeset of a uniform group of rank 2 has at most two elements.

I do not have an example of a uniform group of finite rank with infinite typeset, but there are uniform groups of infinite rank having an infinite typeset, as shown in the following example.

EXAMPLE. Let $\mathscr{S}$ be the rational group with 1 and $\chi^{\mathscr{S}}(1)=(1,1, \ldots) \epsilon$ $t$, let $B=\bigoplus_{i=1}^{\infty} \mathscr{S} x_{i} \subset \bigoplus_{i=1}^{\infty} \mathbf{Q} x_{i}$. Let $P_{i}$ for all natural numbers $i$ be pairwise disjoint infinite sets of primes with union $\bigcup_{i \in \mathrm{N}} P_{i}$ equal to the set of all primes. Let $S_{i}=\left\langle p^{-1} \mid p \in P_{i}\right\rangle \subset \mathbf{Q}$ be rational groups of type $t_{i}=t\left(S_{i}\right)$. Let

$$
A=B+\sum_{i=1}^{\infty} S_{i}\left(y+x_{i}\right) \subset \mathbf{Q} y \oplus \bigoplus_{i=1}^{\infty} \mathbf{Q} x_{i} .
$$

We have using the hypothesis on $P_{i}$ and $S_{i}$, that $B$ and all $S_{i}\left(y+x_{i}\right)$ are pure in $A$ and $A / B \cong \sum_{i \in \mathbf{N}} S_{i} \subset \mathbf{Q}$ is the sum of all the $S_{i}$. Hence as in the remark preceding [M2; 2.1] we have $t(A / B)=t(\mathscr{S})$ and $A$ is $t$-uniform. All types $t_{i}$ are in the typeset of $A$, thus $A$ has an infinite typeset.

Parts of the following proposition were proved by Gardner [G].

Proposition 2.3. If all chief quotients of a composition sequence of a torsion-free abelian group are p-divisible, then the whole group is p-divisible.

For idempotent type $t$ all $t$-uniform groups $A$ satisfy $A=A(t)$ and are completely decomposable homogeneous of type $t$.

Proof. We prove the Proposition by transfinite induction. If $\left(A_{\beta} \mid \beta<\alpha\right)$ is a composition sequence, with $p$-divisible quotients then $A_{i}$ is a $p$-divisible rational group. If $\beta$ is a limit ordinal, then $A_{\beta}=\bigcup_{i<\beta} A_{i}$ is $p$-divisible as a union of $p$-divisible subgroups $A_{i}$. If $i$ is not a limit ordinal, then it is enough to show that $A_{i}$ is $p$-divisible if $A_{i-1}$ is $p$-divisible and $A_{i} / A_{i-1}$ is $p$-divisible. 
Let $x \in A_{i} \backslash A_{i-1}$. Now $A_{i} / A_{i-1}$ is $p$-divisible and there is an element $y$ with $p y-x=z \in A_{i-1}$. But $A_{i-1}$ is $p$-divisible, hence $x$ is divisible by $p$. With $x$ also $p^{-1} x$ is not in $A_{i-1}$ and $x$ is $p$-divisible. Thus $A_{i}$ is $p$-divisible.

If the type $t$ is idempotent and the group is $t$-uniform, then obviously $A=$ $A(t)$. Hence $A$ is homogeneous and completely decomposable by Theorem 2.1 .

LEMMA 2.4. Let $A$ be a t-uniform group with proper homogeneous pure subgroup $B$ of type $t$. If $B$ is of finite rank, then $A / B$ is t-uniform.

Proof. Let a $t$-uniform composition sequence $0 \subset_{*} A_{1} \subset_{*} A_{2} \subset_{*} \cdots \subset_{*} A$ of $A$ be given. Then

$$
0 \subset\left(A_{1}+B\right) / B \subset\left(A_{2}+B\right) / B \subset \cdots \subset A / B
$$

is a sequence with possibly torsion-quotients. We have

$$
\frac{A_{i+1}+B}{A_{i}+B} \cong \frac{A_{i+1} / A_{i}}{\left(\left(A_{i+1} \cap B\right)+A_{i}\right) / A_{i}}
$$

and these quotients are either $\cong A_{i+1} / A_{i}$ if $A_{i+1} \cap B=A_{i} \cap B$ or finite otherwise, because $A_{i+1} \cap B$ is homogeneous of type $t$ as a pure subgroup of the homogeneous group $B$. The group $B$ is of finite rank and $A_{i+1} \cap B \neq$ $A_{i} \cap B$ happens only for finitely many $i$. Forming the sequence $\left\langle A_{i}+B\right\rangle_{*}^{A}$ and observing that $\left\langle A_{i}+B\right\rangle_{*} /\left(A_{i}+B\right)$ is always finite we get (omitting equations) the $t$-uniform composition sequence $\left(\left\langle A_{i}+B\right\rangle_{*}^{A} \mid i<\alpha\right)$ of $A / B$. This proves that $A / B$ is $t$-uniform.

The quotient of a $t$-uniform group relative to a homogeneous pure subgroup of type $t$ is in general not $t$-uniform as the following example shows. Let $A=\bigoplus_{i=0}^{\infty} \mathrm{Z} x_{i}$ be a free group of countable rank. Let $p_{1}, p_{2}, \ldots$ denote the sequence of all primes. The subgroup $B=\bigoplus_{i=1}^{\infty} \mathrm{Z}\left(x_{0}-p_{i} x_{i}\right)$ is pure in $A$ and $A / B$ is a rational group of type $(1,1, \ldots) \in t$, hence $A / B$ is not $t(\mathbf{Z})$-uniform.

Proposition 2.5. Homogeneous pure subgroups of type $t$ int-uniform groups are completely decomposable (homogeneous of type $t$ ).

Proof. If $0 \subset_{*} A_{1} \subset_{*} A_{2} \subset_{*} \cdots \subset_{*} A$ is a $t$-uniform composition sequence of $A$ and $B$ a pure subgroup, then the intersection of this composition sequence with $B$ leads to a sequence of pure subgroups

$$
0 \subset A_{1} \cap B \subset A_{2} \cap B \subset \cdots \subset A \cap B=B
$$


with possible equalities. We have $\left(A_{i+1} \cap B\right) /\left(A_{i} \cap B\right) \tilde{\subset} A_{i+1} / A_{i}$ and the quotients are either 0 or of type $\leq t$. But $B$ was homogeneous and pure, hence elements in $\left(A_{i+1} \cap B\right) \backslash\left(A_{i} \cap B\right)$ have type $t$. Thus $B$ is $t$-uniform, and completely decomposable by 2.1 .

Proposition 2.6. All t-radical subgroups of finite rank in $t$-uniform groups are t-uniform.

Proof. Let $B \subset A$, where $B$ is $t$-radical and $A$ t-uniform. By 1.2 it is enough to show that the pure hull of $B$ is $t$-uniform. So we may assume $B$ to be pure. We prove this by induction. The start and assumption are obvious. If for $n>1$

$$
0=B_{0} \subset_{*} B_{1} \subset_{*} B_{2} \subset_{*} \cdots \subset_{*} B_{n}=B
$$

is a composition chain of $B$ with types $t_{i}=t\left(B_{i} / B_{i-1}\right) \geq t$, then $B / B_{1}$ is $t$-radical by 1.3 and $A / B_{1}$ is $t$-uniform by 2.4 . Moreover, $t \leq t\left(B_{1}\right) \leq t$ by definition of $t$-radical groups and by 2.2 using that $B_{1}$ is a pure subgroup of the $t$-uniform group $A$. Thus the hypothesis can be applied to prove that $B$ is $t$-uniform.

If $t=t(Z)$, then $t$-uniform groups are homogeneous and therefore free by 2.1. Hence the $t$-radical Baer-Specker group cannot be a subgroup of any $t$-uniform group. But this case is too special to decide if there is a $t$ uniform group having a proper $t$-radical subgroup. Further if $\left(B_{i} \mid i \in \mathbf{N}\right)$ is a composition sequence of the pure $t$-radical subgroup $B=\bigcup_{i=1}^{\infty} B_{i}$ of the $t$-uniform group $A$, then $B$ is a $t$-uniform and of countable rank. This can be shown inductively. $B_{1}$ is a rational group of type $t$, that is, a pure homogeneous subgroup of rank 1 in $A$, because $t\left(B_{1}\right) \geq t$ and $t$-uniform groups have only elements of type $\leq t$ by 2.2 . Thus $B / B_{1}$ is a pure $t$ radical subgroup of the quotient $A / B_{1}$, which is $t$-uniform by 2.4 . The same argument applies to all $B_{i+1} / B_{i}$, which are all of type $t$ and $B$ is $t$ uniform. I do not have an example of a $t$-uniform group with (for instance countable) proper $t$-radical subgroup.

A torsion-free abelian group is said to be locally $t$-radical, if all pure subgroups of finite rank are $t$-radical. These are precisely the $t$-radical groups such that all elements have type $\geq t$. Similarly we define groups to be locally $t$-uniform. Locally $t$-uniform groups are always homogeneous of type $t$ and they are completely decomposable if of finite rank, that is, locally $t$-uniform groups are precisely the locally (completely decomposable homogeneous of type $t$ )-groups. 
The following result shows that the Baer-Specker group is a locally $t$ uniform group for $t=t(Z)$ and of smallest cardinality, which is not $t$ uniform.

Proposition 2.7. Countable locally t-uniform groups are completely decomposable homogeneous of type $t$.

Proof. Let $x_{1}, x_{2}, \ldots$ be an indexing of the elements of the countable locally $t$-uniform group $A$. If we select a composition sequence $\left(A_{i} \mid i \in \mathbf{N}\right)$ out of

$$
\left\langle x_{1}\right\rangle_{*} \subset_{*}\left\langle x_{1}, x_{2}\right\rangle_{*} \subset_{*}\left\langle x_{1}, x_{2}, x_{3}\right\rangle \subset_{*} \ldots
$$

omitting equalities, then $A_{i}$ is completely decomposable homogeneous of type $t$ by 2.1 , and $A_{i-1}$ is a direct summand of $A_{i}$ with $A_{i} / A_{i-1}$ of type $t$. By the same argument as in the proof of 2.1 we get $A=\bigcup_{i \in \mathrm{N}} A_{i}$ to be completely decomposable.

\section{Radical groups of finite rank, Butler groups}

We consider uniform and radical groups of finite rank.

TheOREM 3.1. If $A$ is a $t$-uniform group of finite rank, then $A=A^{\infty}(t)$, $A / A^{i}(t)$ is $t$-uniform and $A^{i+1}(t) / A^{i}(t)$ is completely decomposable homogeneous of type $t$ for all $i$. If the type $t$ is idempotent, then $A$ is completely decomposable homogeneous of type $t$.

Proof. By finiteness of the rank we have only to prove that $A / A(t)$ is a $t$-uniform group and $A(t)$ is completely decomposable homogeneous of type $t$. Since $A$ is $t$-uniform, by 2.2 all elements have type $\leq t$, thus $A(t)$ is homogeneous of type $t$ and completely decomposable by 2.5 . Hence $A / A(t)$ is $t$-uniform by 2.4 .

If $t$ is idempotent, then $A$ is completely decomposable homogeneous of type $t$ by 2.3 .

Theorem 3.1 can only be proved for groups of finite rank. If $A$ is a $t$ uniform group, then $A / A(t)$ is $t$-radical by 1.3 but need not be $t$-uniform as the following example shows. Let $p_{1}, p_{2}, \ldots$ be the sequence of all primes. Let $S$ be the rational group with 1 and $\chi^{S}(1)=(1,1, \ldots) \in t$. Let $B=$ $\bigoplus_{i=1}^{\infty} S x_{i} \subset \bigoplus_{i=1}^{\infty} Q x_{i}$ and

$$
A=\left\langle B, p_{i}^{-2}\left(x_{2 i-1}+p_{i} y\right), p_{2 i}^{-2}\left(x_{2 i}+y\right) \mid i \in \mathbf{N}\right\rangle \subset \mathbf{Q} y \oplus \bigoplus_{i=1}^{\infty} \mathbf{Q} x_{i} \text {. }
$$


Then $A$ has the $t$-uniform composition sequence

$$
\begin{aligned}
& 0 \subset_{*}\left\langle x_{1}\right\rangle_{*} \subset_{*}\left\langle x_{1}, x_{3}\right\rangle_{*} \subset_{*} \cdots \subset_{*} \bigoplus_{i=1}^{\infty} S x_{2 i-1} \\
& \quad=: C \subset_{*}\langle C, y\rangle_{*} \subset_{*}\left\langle C, y, x_{2}\right\rangle_{*} \subset_{*} \cdots \subset_{*} A .
\end{aligned}
$$

But $A(t)=B$ since $(0,1,0,1, \ldots) \in t(y)$, and

$$
A / B=\langle y+B\rangle_{*}, \quad(1,2,1,2, \ldots) \in t(y+B) .
$$

Thus the rational group $A / A(t)$ is not of type $t$, hence not $t$-uniform.

Butler groups, that is, pure subgroups of completely decomposable groups of finite rank (compare [A]), turn out to be very special if they satisfy certain type conditions.

LEMMA 3.2. A Butler group with type sequence $\left(t_{i} \mid 1 \leq i \leq n\right)$ is completely decomposable if for all $i<n$ there is $a j>i$ with $\bigcap_{k=1}^{i} t_{k} \geq t_{j}$.

Proof. We prove by induction on $n$ that a Butler group with the given type chain is isomorphic to $\bigoplus_{i=1}^{n} A_{i}$ where $t\left(A_{i}\right)=t_{i}$. The start and assumption are obvious. Let $A$ be a group of rank $n$. Let $B$ be a pure subgroup with type chain $\left(t_{i} \mid 1 \leq i \leq n-1\right)$ and quotient $A / B$ of type $t_{n}$. Then $B$ is completely decomposable of inner type $I T(B)=\bigcap_{i=1}^{n-1} t_{i}$ by hypothesis. Consequently $I T(A)=\bigcap_{i=1}^{n-1} t_{i} \cap t(x)=t(x)$ for all $x \in A \backslash B$, because $t(x) \leq t(x+B)=t_{n} \leq \bigcap_{i=1}^{n-1} t_{i}$. Hence all elements in $A \backslash B$ have the same type say $s$.

Now a Butler group can be written in the form $A=\sum_{i=1}^{n} A_{i}$ with pure rational groups $A_{i}$ and $A / B=\sum\left\{\left(A_{i}+B\right) / B \mid A_{i} \not \subset B\right\}$ is of type $t_{n}=$ $t(A / B)=\bigcup\left\{t\left(A_{i}\right) \mid A_{i} \not \subset B\right\}=s$. We have using Baer's Lemma [F; 86.5] that $B$ is a direct summand and $A$ is completely decomposable of the desired isomorphism type.

COROLLARY 3.3 ([A; 1.11]). A Butler group with linearly ordered typeset is completely decomposable.

COROLlaRY 3.4. For a $t$-uniform group $A$ of finite rank the following are equivalent:

(1) $A$ is a Butler group;

(2) $A$ is homogeneous of type $t$;

(3) $A$ is completely decomposable homogeneous of type $t$.

Proof. By 2.1 (2) and (3) are equivalent. That (3) implies (1) is trivial and (1) implies (3) by 3.2 . 
THEOREM 3.5. The t-radical of a Butler group $A$ equals the type subgroup $A(t)$ for all types $t$, and a Butler group is $t$-radical if and only if $t$ is the inner type.

Proof. To prove the first statement it is enough to show that for a Butler group $A$ the quotient $A / A(t)$ cannot be rational of type $\geq t$. The Butler group $A$ is a finite sum of rational subgroups, that is $A=A(t)+\sum_{i=1}^{k} A_{i}$, where the $A_{i}$ are rational and $A_{i} \cap A(t)=0$ for all $i$. We may assume by finiteness of the typeset of a Butler group that the type $t$ is realized. Assume $t(A / A(t)) \geq t$. We have

$$
A / A(t)=\sum_{i=1}^{k}\left(A_{i} \oplus A(t)\right) / A(t)
$$

is the sum of rational groups with types $t\left(A_{i}\right)$, that is, $t(A / A(t))=\bigcup_{i=1}^{k} t\left(A_{i}\right)$ $\geq t$. The inner type of $A(t)$ is $t$ and for the inner type of $A$ we have

$$
t \geq I T(A)=I T(A(t)) \cap t\left(A_{i}\right)=t \cap t\left(A_{i}\right)
$$

for all $i$. None of the types $t\left(A_{i}\right)$ can be $\geq t$ because $A(t) \neq A$. But now we get the contradiction

$$
t=t \cap \bigcup_{i=1}^{k} t\left(A_{i}\right)=\bigcup_{i=1}^{k}\left(t \cap t\left(A_{i}\right)\right)=I T(A)<t .
$$

By the finiteness of the typeset of a Butler group the inner type is realized [M2; 1.3]. Thus a Butler group is $t$-radical if and only if $t$ is the inner type.

All homogeneous torsion-free abelian groups of finite rank and not only Butler groups have the property of Theorem 3.5, but Butler groups are, excluding the trivial homogeneous case with 3.4 , not homogeneous.

REMARK. A torsion-free abelian group is called quotient-divisible if there is a free subgroup such that the quotient is the direct sum of a divisible group and a group of finite exponent. A $t$-uniform group of finite rank is quotient-divisible if and only if $t$ is idempotent, because $t$ is also a cotype and torsion-free homomorphic images of quotient-divisible groups are again quotient-divisible and a rational group is quotient-divisible if and only if it is of idempotent type. Thus a $t$-uniform quotient-divisible group of finite rank is completely decomposable homogeneous of type $t$ by 3.1 .

\section{Quasi-decompositions}

If $A_{i}, i<\alpha$, are subgroups of a torsion-free abelian group $A$ which form their direct sum, then $\bigoplus_{i<\alpha} A_{i}$ is said to be a quasi-decomposition 
of $A$ if the quotient $A /\left(\bigoplus_{i<\alpha} A_{i}\right)$ has finite exponent. If $A$ has the quasidecomposition $\bigoplus_{i<\alpha} A_{i}$ where $A_{i}$ is a $t_{i}$-uniform group with type sequence of length $n_{i}$, which is an ordinal, then all permutations of the type sequence $\left(t_{1}^{n_{1}}, t_{2}^{n_{2}}, \ldots, t_{i}^{n_{i}}, \ldots\right)$ are also type sequences of $A$. With Theorem 4.3 we prove a result in the opposite direction. But there are torsion-free abelian groups having this permutation property but no quasi-decomposition into $t_{i}$ uniform subgroups if there are either infinitely many pairwise different types $t_{i}$ or if at least two of the ordinals $n_{i}$ are infinite. This is shown by the following two examples.

Let $P_{0}, P_{1}, \ldots$ be infinitely many pairwise disjoint infinite sets of primes. Let $S_{i}=\left\langle p^{-1} \mid p \in P_{i}\right\rangle \subset \mathbf{Q}$ and $t_{i}=t\left(S_{i}\right)$. Let $B=\bigoplus_{i=1}^{\infty} S_{i} x_{i} \subset \bigoplus_{i=1}^{\infty} \mathbf{Q} x_{i}$ and

$$
A=\left\langle B,\left(x_{i}+x_{i+1}\right) / q_{i} \mid 1 \leq i<\infty\right\rangle,
$$

where $q_{1}, q_{2}, \ldots$ is an indexing of $P_{0}$. Let $A_{i}=\left\langle x_{1}, x_{2}, \ldots, x_{i}\right\rangle_{*}^{A}$, then $\left(A_{i} \mid 1 \leq i<\infty\right)$ is a composition sequence of $A$ with corresponding type sequence $\left(t_{i} \mid 1 \leq i<\infty\right)$. All permutations of this type sequence are again type sequences of $A$. We have $t_{i} \cap t_{j}=t(\mathrm{Z})$ if $i \neq j$ and therefore $A^{\infty}\left(t_{i}\right)=A\left(t_{i}\right)=S_{i} x_{i}$. Thus $B$ is a maximal subgroup of $A$ having a direct decomposition into $t_{i}$-uniform subgroups. But $A / B \cong \bigoplus_{i=1}^{\infty} \mathbf{Z}\left(q_{i}\right)$ is not of finite exponent and $B=\bigoplus_{i=1}^{\infty} S_{i} x_{i}$ is not a quasi-decomposition of A.

Another example $D$ is given by

$$
c=\bigoplus_{i=1}^{\infty} S_{1} x_{i} \oplus \bigoplus_{i=1}^{\infty} S_{2} y_{i} \subset \bigoplus_{i=1}^{\infty} \mathbf{Q} x_{i} \oplus \bigoplus_{i=1}^{\infty} \mathbf{Q} y_{i}
$$

and $D=\left\langle C,\left(x_{i}+y_{i}\right) / q_{i} \mid 1 \leq i<\infty\right\rangle$. Obviously $D$ has the permutation property for the type sequence $\left(t_{1}^{\omega}, t_{2}^{\omega}\right)$. By $D^{\infty}\left(t_{1}\right)=\bigoplus_{i=1}^{\infty} S_{1} x_{i}$ and $D^{\infty}\left(t_{2}\right)=\bigoplus_{i=1}^{\infty} S_{2} y_{i}$ we conclude that $C$ is a maximal subgroup of $D$ having a direct decomposition into $t_{i}$-uniform subgroups. But $D / C \cong \bigoplus_{i=1}^{\infty} \mathbf{Z}\left(q_{i}\right)$ is not of finite exponent and $C$ is not a quasi-decomposition of $A$.

If a subgroup $B$ of a torsion-free abelian group $A$ of finite rank has a type chain which is also a type chain of $A$, then certainly $A / B$ is a reduced torsion group by [M2;3.4], because $A$ and $B$ have equal sum type, but $A / B$ is not necessarily finite, nor even of finite exponent [M2; Example B following 3.6].

LemMA 4.1. Let $A$ be a torsion-free abelian group of finite rank $m$ with subgroup $B$ of the same rank $m$. If $A$ and $B$ have the same type chain $\left(t_{1}^{n_{1}}, \ldots, t_{k}^{n_{k}}\right)$ with pairwise different types $t_{i}$, where $t_{i}$ is always a maximal 
element in the (partially ordered) set $\left\{t_{i}, t_{i+1}, \ldots, t_{k}\right\}$ for $1 \leq i \leq k$, then $A / B$ is finite.

Proof. Let $\left(A_{i} \mid 1 \leq i \leq m\right)$ and $\left(B_{i} \mid 1 \leq i \leq m\right)$ be composition chains corresponding to the given type chain of $A$ and $B$ respectively. Let $t=t_{1}$ and $n=n_{1}$. By 1.4 we have $A_{n}, B_{n} \subset A^{\infty}(t)$. Assuming $A_{n} \neq A^{\infty}(t)$ we get $0 \neq A^{\infty}(t) / A_{n} \subset A / A_{n}$, that is, $A / A_{n}$ contains an element of type $\geq t$ by 1.3 . This is a contradiction by 2.2 and the maximality of $t$. So we have $A_{n}=A^{\infty}(t)$ and $B_{n}=B^{\infty}(t)$ and both are $t$-uniform groups. Further we have $0 \neq B(t) \subset A(t)$. By 2.5 both type subgroups are completely decomposable homogeneous of type $t$. The group $B(t)_{*}^{A}$ is completely decomposable homogeneous of type $t$ as a pure subgroup of the completely decomposable homogeneous group $A(t)$ by [F; 86.6]. To show that $B(t)_{*}^{A} / B(t)$ is finite we could use a modification of [F;98.1] or observe that $O T\left(B(t)_{*}^{A}\right)=I T\left(B(t)_{*}^{A}\right)=t$ and $S T\left(B(t)_{*}^{A}\right)=S T(B(t))=n t$ and apply [M2; 3.6]. Consequently $\left(B+B(t)_{*}^{A}\right) / B \cong B(t)_{*}^{A} / B(t)$ is finite and we may assume $B(t)$ to be pure in $A$.

Now we prove the statement by induction on the rank $m$ of $A$. When $m=1$ the result is clear. If $B(t)$ is assumed to be pure in $A$, then $B(t) \subset A_{n}$ and $B(t) \subset B_{n}$. The quotients $A_{n} / B(t)$ and $B_{n} / B(t)$ are $t$-uniform by 2.4 . But then for the groups $A / B(t)$ and $B / B(t)$ the hypothesis applies and $A / B \cong(A / B(t)) /(B / B(t))$ is finite.

Let $A=\bigoplus_{i=1}^{\infty} \mathbf{Z} x_{i}$ be free with subgroup $B=\bigoplus_{i=1}^{\infty} \mathbf{Z} p_{i} x_{i}$, where $p_{1}$, $p_{2}, \ldots$ is an indexing of the set of all primes, so $A / B$ does not have finite exponent. But $A$ and $B$ are free and have both only one (and the same) type sequence. Thus 4.1 cannot be proved for groups of infinite rank.

COROLlary 4.2. In a $t$-uniform group of finite rank all $t$-uniform subgroups with torsion quotient are of finite index.

In view of two examples $A$ and $D$ above it is natural in this context of quasi-decompositions to restrict attention to torsion-free abelian groups having a type sequence $\left(t_{1}^{n_{1}}, \ldots, t_{k}^{n_{k}}\right)$, where only finitely many different types occur and only one of these types occurs infinitely often, that is, only one of the numbers $n_{i}$ is possibly an infinite ordinal. The following result fills the gap left by these two examples.

THEOREM 4.3. Let $A$ be a torsion-free abelian group with type sequence $\left(t_{1}^{n_{1}}, \ldots, t_{k}^{n_{k}}\right)$, where the types $t_{1}, \ldots, t_{k}$ are pairwise different, and at most one of the numbers $n_{i}$ is infinite. If all block-permutations $\left(t_{\pi(1)}^{n_{\pi(1)}}, \ldots, t_{\pi(k)}^{n_{\pi(k)}}\right)$, 
$\pi$ a permutation, are also type sequences of $A$, then $A$ has a subgroup $\bigoplus_{i=1}^{k} A_{i}$ of finite index, where the $A_{i}$ are pure $t_{i}$-uniform subgroups of rank $n_{i}$.

In particular $A$ has then all permutations of the given type sequence as type sequences.

The $t$-radical of $A$ is $\left\langle\bigoplus_{t_{i} \geq t} A_{i}\right\rangle_{*}$.

Proof. Assume $t=t_{1}$ to be a maximal type among the $t_{1}, \ldots, t_{k}$. Observe that the types $t_{1}, \ldots, t_{k}$ are not assumed to be linearly ordered. Let $B$ be a $t$-uniform pure subgroup of rank $n=n_{1}$, possibly an infinite cardinal, belonging to a composition sequence corresponding to the type sequence $\left(t_{1}^{n_{1}}, \ldots, t_{k}^{n_{k}}\right)$. By $1.4 B$ is contained in the $t$-radical $A^{\infty}(t)$ of $A$. Moreover $A^{\infty}(t) / B$ is a $t$-radical group if not 0 by 1.3. If $A^{\infty}(t) \neq B$, then $A / B$ contains an element with type $\geq t$. By 2.2 any type sequence of $A / B$, for instance $\left(t_{2}^{n_{2}}, \ldots, t_{k}^{n_{k}}\right)$ must contain a type $\geq t$. But $T$ was maximal and the $t_{i}$ were pairwise different. Thus we have a contradiction. Hence $A^{\infty}(t)=B$. Consequently all composition sequences, starting with $n$-times $t$, have to meet $A^{\infty}(t)$ and therefore $A / A^{\infty}(t)$ has the block-permutation property for the type sequence $\left(t_{2}^{n_{2}}, \ldots, t_{k}^{n_{k}}\right)$.

Let $C$ be a pure subgroup of $A$ with type sequence $\left(t_{2}^{n_{2}}, \ldots, t_{k}^{n_{k}}\right)$ and $t_{1}$-uniform quotient $A / C$ of rank $n_{1}$. Now we prove $C \cap A^{i}(t)=0$ by induction on $i$. Using 2.2 and the maximality of $t$ we have $C \cap A^{1}(t)=0$. Now we assume $C \cap A^{i}(t)=0$. Let $\bar{A}=A / A^{i}(t), \bar{C}=\left(C \oplus A^{i}(t)\right) / A^{i}(t) \cong C$ and $\bar{A}(t)=\left(A / A^{i}(t)\right)(t)$. Again by 2.2 we have $\bar{C} \cap \bar{A}(t)=0$ but $\bar{A}(t)=$ $A^{i+1}(t) / A^{i}(t)$ and consequently by the modular law

$$
\left(C \cap A^{i+1}(t)\right) \oplus A^{i}(t)=\left(C \oplus A^{i}(t)\right) \cap A^{i+1}(t)=A^{i}(t),
$$

that is $C \cap A^{i+1}(t)=0$. If $\alpha$ is a limit ordinal and $C \cap A^{i}(t)=0$ for all $i<\alpha$, then obviously

$$
C \cap A^{\alpha}(t)=C \cap \bigcup_{i<\alpha} A^{i}(t)=0 .
$$

This shows that $C \cap A^{\infty}(t)=0$.

Now we prove the theorem by induction on the number $k$ of different types. The case $k=1$ and the hypothesis are clear. Let $k>1$. By the considerations above and the hypothesis, $A / A^{\infty}(t)$ has the decomposable subgroup $\bigoplus_{i=2}^{k} \bar{A}_{i}$ of finite index, with $t_{i}$-uniform groups $\bar{A}_{i}$ of rank $n_{i}$.

If $A^{\infty}(t)$ is of infinite rank, that is, $n_{1}$ is infinite, then

$$
A / A^{\infty}(t) \supset\left(C \oplus A^{\infty}(t)\right) / A^{\infty}(t) \cong C,
$$


are both groups of finite rank, and the groups $\left(C \oplus A^{\infty}(t)\right) / A^{\infty}(t)$ and $C$ have equal type chains of the kind used in 4.1 , if we start with an indexing $t_{1}, \ldots, t_{k}$ such that $t_{i}$ is maximal in $\left\{t_{i}, \ldots, t_{k}\right\}$ for all $i$. Hence $C \oplus$ $A^{\infty}(t)$ has finite index in $A$ and $A^{\infty}(t)$ is a quasi-summand. Further $C$ is quasi-isomorphic to $A / A^{\infty}(t)$ and has therefore a quasi-decomposition $\bigoplus_{i=2}^{k} A_{i}$ like $A / A^{\infty}(t)$ with pure $t_{i}$-uniform subgroups $A_{i} \cong \bar{A}_{i}$ and with $A_{1}=A^{\infty}(t)$ the sum $\bigoplus_{i=1}^{k} A_{i}$ is the desired decomposable subgroup of $A$ of finite index.

If $A^{\infty}(t)$ has finite rank, then

$$
A / C \supset\left(A^{\infty}(t) \oplus C\right) / C \cong A^{\infty}(t) .
$$

$A / C$ and $A^{\infty}(t)$ are $t$-uniform groups of finite rank and consequently $A^{\infty}(t)$ $\oplus C$ is of finite index in $A$ by 4.2. Thus $A / A^{\infty}(t)$ contains a subgroup isomorphic to $C$ of finite index. But subgroups of finite index have the same type sequences as the whole group. Therefore $C$ has the permutation property too. By hypothesis $C$ has a decomposable subgroup $\bigoplus_{i=2}^{k} A_{i}$ of finite index and again $\bigoplus_{i=1}^{k} A_{i}$ with $A_{1}=A^{\infty}(t)$ is the desired decomposable subgroup of finite index. If $t$ is an arbitrary type and $D=\left\langle\sum_{t_{i} \geq t} A_{i}\right\rangle_{*}$, then by $1.4, D \subset A^{\infty}(t)$ and $A^{\infty}(t) / D$ is a $t$-radical group by 1.3. Now $\bigoplus_{t_{i} x_{t}} A_{i}$ is isomorphic to a subgroup of finite index in $A / D \supset A^{\infty}(t) / D$. Thus $A^{\infty}(t)=D$ by 2.2 .

By Theorem 4.3 the existence of all block-permutations imply the existence of all permutations of a type chain in case of finite rank.

COROLLARY 4.4. A torsion-free abelian group of finite rank, having one type chain together with all permutations, has a quasi-decomposition into uniform groups.

An immediate consequence is the following unpublished result of Burkhardt (1982).

COROLlaRY 4.5 (Burkhardt). Let $t_{1}, \ldots, t_{k}$ be pairwise different types. If the torsion-free abelian group $A$ of finite rank $k$ has the type chain $\left(t_{1}, \ldots, t_{k}\right)$ together with all permutations, then $A$ is almost completely decomposable with quasi-decomposition $\bigoplus_{i=1}^{k} A_{i}$, where $A_{i}$ is a rational group of type $t_{i}$.

CoRollaRy 4.6. A Butler group having a type chain together with all permutations is almost completely decomposable.

Proof. This is a consequence of 4.4 and 3.4. 
Example $\mathbf{E}$ is a $t$-uniform group, where $(1,1, \ldots) \in t$, that is a group having a type chain together with all permutations. But $\mathbf{E}$ is not a Butler group.

COROLLARY 4.7. A torsion-free abelian group having a type sequence of only idempotent types together with all permutations is almost completely decomposable if only finitely many different types occur and at most one of these types occurs infinitely often.

Proof. This is a consequence of 4.3 and 2.3.

Corollary 4.8. A quotient-divisible group of finite rank, having a type chain together with all permutations, is almost completely decomposable.

Proof. If a type chain $\left(t_{i} \mid i\right)$ occurs with all permutations, then all types $t_{i}$ are cotypes. But cotypes of quotient-divisible groups are idempotent (compare the last remark in Section 3). The rest follows by 4.7.

\section{Acknowledgement}

I thank the referee and K. M. Rangaswamy for many helpful remarks.

\section{References}

[A] D. M. Arnold, Pure subgroups of finite rank completely decomposable groups, (Lecture Notes in Math. 874, pp. 1-31, Springer-Verlag, 1981).

[F] L. Fuchs, Infinite abelian groups I + II , (Academic Press, 1970, 1973).

[G] B. J. Gardner, 'A note on types,' Bull. Austral. Math. Soc. 2 (1970), 275-276.

[M1] O. Mutzbauer, Type graph, (Lecture Notes in Math. 1006, pp. 228-252, Springer-Verlag, 1983).

[M2] O. Mutzbauer, 'Type invariants of torsion-free abelian groups,' Abelian Group Theory Proc. Perth, 1987, pp. 133-153, (Contemp. Math. Vol. 87, 1989).

[R] D. J. S. Robinson, Finiteness conditions and generalized soluble groups, Part 1 (SpringerVerlag, 1972).

[S] P. Schultz, 'Annihilator classes of torsion-free abelian groups,' Topics in Algebra, Proc. Canberra (Lecture Notes in Math. 697, Springer-Verlag, 1978).

Universität Würzburg

Mathematisches Institut

Am Hubland

8700 Würzburg

Bundesrepublik Deutschland 